\title{
Assessing the risk of alcohol-induced dose dumping from sustained-release oral dosage forms: in vitro-in silico approach
}

\author{
Sandra Cvijić, Ivana Aleksić, Svetlana Ibrić \& Jelena Parojčić
}

To cite this article: Sandra Cvijić, Ivana Aleksić, Svetlana Ibrić \& Jelena Parojčić (2017): Assessing the risk of alcohol-induced dose dumping from sustained-release oral dosage forms: in vitro-in silico approach, Pharmaceutical Development and Technology, DOI: 10.1080/10837450.2017.1392973

To link to this article: http://dx.doi.org/10.1080/10837450.2017.1392973

Accepted author version posted online: 18 Oct 2017.

Submit your article to this journal

WII Article views: 1

Q View related articles $\sqsubset$

View Crossmark data [ 


\section{Assessing the risk of alcohol-induced dose dumping from sustained- release oral dosage forms: in vitro-in silico approach}

Sandra Cvijić, Ivana Aleksić, Svetlana Ibrić, Jelena Parojčić

Department of Pharmaceutical Technology and Cosmetology, University of Belgrade Faculty of Pharmacy, Belgrade, Serbia

Corresponding author:

Sandra Cvijić

Department of Pharmaceutical Technology and Cosmetology

University of Belgrade - Faculty of Pharmacy

Vojvode Stepe 450

11221 Belgrade, Serbia

Tel: +381113951356

Fax: +381113972840

E-mail address: gsandra@ pharmacy.bg.ac.rs

Ivana Aleksić: Tel: +381113951356; E-mail: ivana.aleksic@ pharmacy.bg.ac.rs

Svetlana Ibrić: Tel: +381113951363; E-mail: ibric@ pharmacy.bg.ac.rs

Jelena Parojčić: : Tel: +381113951363; E-mail: jelena.parojcic@ pharmacy.bg.ac.rs 


\section{Assessing the risk of alcohol-induced dose dumping from sustained- release oral dosage forms: in vitro-in silico approach}

Consumption of alcoholic beverages with sustained-release oral dosage forms may pose a risk to patients due to potential alcohol-induced dose dumping (ADD). Regulatory guidances recommend in vitro dissolution testing to identify the risk of $\mathrm{ADD}$, but the question remains whether currently proposed test conditions can be considered biopredictive. The purpose of this study was to evaluate different dissolution setups to assess ADD, and the potential of combined in vitro-in silico approach to predict drug absorption after concomitant alcohol intake for hydrophilic and lipophilic sustained-release tablets containing ibuprofen or diclofenac sodium. According to the obtained results, the impact of ethanol was predominantly governed by the influence on matrix integrity, with the increase in drug solubility being less significant. Hydrophilic matrix tablets were less susceptible to ADD than lipophilic matrices, although the conclusion on formulation ethanol-vulnerability depended on the employed experimental conditions. In silico predictions indicated that the observed changes in drug dissolution would not result in plasma concentrations beyond therapeutic window, but sustained-release characteristics of the formulations might be lost. Overall, the study demonstrated that in vitro-in silico approach may provide insight into the effect of ADD on drug clinical performance, and serve as a tool for ADD risk assessment.

Keywords: alcohol-induced dose dumping; sustained release; dissolution; physiologically-based modeling; hydrophilic matrix tablets; lipophilic matrix tablets

\section{Introduction}

Alcohol-induced dose dumping (ADD) is a potentially dangerous phenomenon that signifies abrupt drug release following concomitant intake of alcoholic beverages and modified (MR), particularly extended (ER) or sustained release (SR) oral dosage forms. As a consequence, systemic drug exposure and therapeutic effect can be significantly altered, thus posing a serious risk to patients. 
The likelihood of ADD for MR products have mostly been overlooked in the past. However, a breakthrough happened in 2005 when the FDA issued the alert on alcohol-Palladone $^{\mathrm{TM}}$ (hydromorphone hydrochloride extended-release capsules; Purdue Pharma, US) interaction (FDA 2005). This product was withdrawn from the US market after a pharmacokinetic study revealed alarming results that co-ingestion of Palladone ${ }^{\mathrm{TM}}$ and alcohol led to significantly higher, and potentially lethal peak plasma concentrations of the drug (i.e. six times greater average peak drug concentration following co-ingestion of $12 \mathrm{mg}$ Palladone capsule with $240 \mathrm{ml}$ of $40 \%$ alcoholic beverage compared to co-ingestion with water). This case pioneered new perspectives on the influence of concomitant alcohol intake on drug release mechanism, and initiated new guidances which put the ADD risk assessment in the focus of formulation development for MR dosage forms. The objective of such an approach has been to promote the design of rugged, alcohol-resistant MR formulations in order to minimize the risk of ADD, even when appropriate warnings are included in a product labelling. Namely, survey data indicate that alcohol consumption in these cases is still very likely, especially with patients who suffer from chronic pain, and tend to consume alcohol to cope with their conditions (Brennan et al. 2005). Moreover, accidental ADD may happen to any patient, because consumption of alcohol beverages is a common habit in most cultures. According to the WHO database, $38.3 \%$ of the world population (15+ years) regularly consumes alcohol, with the highest consumption levels in European region and America (WHO 2014).

It has been recognized that increased solubility of drugs or excipients in the presence of ethanol, and/or formulation factors such as inhibition of polymer swelling or matrix dissolution, in conjunction with changes in physiological conditions after alcohol intake (e.g. delayed gastric emptying) are the key factors affecting the kinetics 
of drug release from MR oral dosage forms (Lennernäs 2009; Jedinger et al. 2014).

However, sensitivity of drug and/or excipients to ethanol does not necessarily mean that a formulation would lose its modified release properties. Drug-excipients-dosage form interactions are rather complex, so the degree of alcohol-sensitivity should be accessed on the case-by-case basis. In addition, the risk in case of ADD should be considered alongside therapeutic indication and drug therapeutic index.

Regulatory guidances provide general recommendations regarding the assessment of ADD, and state that suitable in vitro dissolution tests in the presence of ethanol should be carried out to identify the risk of ADD, and differentiate between rugged and vulnerable formulations (Meyer \& Hussain 2005; Anand et al. 2011; FDA 2011; EMA 2014; FDA 2014b; EMA 2016; FDA 2016b). In addition, the International Pharmaceutical Excipients Council Europe (IPEC) working group on ADD has summarized relevant scientific and regulatory information (Friebe 2015; Friebe et al. 2015) to help pharmaceutical companies to better handle ADD issues. The main problem for the formulators is that the recommendations regarding ADD assessment in EU and US guidances are not fully harmonised. For example, EMA mandates ADD testing for all oral MR dosage forms, while FDA has confined this requirement solely for drugs with a narrow therapeutic index (FDA 2008; FDA 2010; FDA 2014a; FDA 2014c; FDA 2014d). Furthermore, there are differences in the recommended dissolution methodology. According to EMA, dissolution medium should be the same as that proposed for routine testing, with 5\%,10\% and $20 \%(\mathrm{v} / \mathrm{v})$ ethanol added, and testing time is not defined. FDA requirements are somewhat more specific, indicating $2 \mathrm{~h}$ testing in $900 \mathrm{ml}$ of $0.1 \mathrm{M} \mathrm{HCl}$ with up to $40 \%$ (v/v) ethanol, with $15 \mathrm{~min}$ sampling interval. These test conditions are selected to emulate the 'worst case' scenario when a dosage form is exposed to a strong alcohol beverage during a sufficient lag-time in 
gastric emptying (Lennernäs 2009). However, recently added FDA Draft Guidance on dexlansoprazole delayed release orally disintegrating tablets states two-stages dissolution testing to assess the risk of ADD: $2 \mathrm{~h}$ in $500 \mathrm{ml} 0.1 \mathrm{~N} \mathrm{HCl}$ without/with 5, 20 and 40\% (v/v) alcohol (basket apparatus, $100 \mathrm{rpm}$ ), followed by testing in $900 \mathrm{ml} 50$ $\mathrm{mmol} / \mathrm{l}$ phosphate buffer (pH 7.2) containing $5 \mathrm{mM}$ sodium lauryl sulfate (SLS), and the same level of ethanol as the acid stage (FDA 2016a). Several research groups also employed in vitro dissolution setups based on 'media change' principle (hydro-alcoholic solutions followed by the relevant non-alcoholic medium) to assess ethanol effect on drug release profile (Levina et al. 2007; Fadda et al. 2008). Still, the overall conclusion of Fadda et al. (2008) was that the extent of ethanol-induced impairment of drug release controlling mechanism is not possible to predict.

There are some opinions that in vitro ADD risk assessment should be carried out under 'more mild' dissolution test conditions (i.e. medium with less than $40 \%$ (v/v) ethanol, less than $2 \mathrm{~h}$ testing in acidic medium) (Friebe 2015). This standpoint is supported by arguments that ethanol is diluted in gastric medium due to gastric secretion and intake of food and liquids, and also ethanol is quickly cleared from the stomach (Levitt et al. 1997; Lennernäs 2009). Another issue on the biorelevancy of the EMA and FDA proposed in vitro test conditions concerns a lack of in vitro-in vivo relationship (IVIVR). This was well illustrated in the study with carvedilol phosphate CR capsules (Coreg $\mathrm{CR}^{\circledR}$, GlaxoSmithKline, US) which failed ADD in vitro dissolution test due to increased solubility of the coating agent (methacrylic copolymers) in ethanol (Henderson et al. 2007). However, drug product in vivo performance was not affected in the presence of alcohol, most likely because stomach residence time and drug exposure to ethanol for multiparticulate dosage form are relatively short. 
A possible way to determine relevance of in vitro dissolution test results to the corresponding pharmacokinetic outcome, while bypassing clinical studies, is through the employment of in silico physiologically-based pharmacokinetic (PBPK) modeling. These sophisticated computational tools have received widespread attention over the past few years, and their potential to forecast clinical implications of ADD have also been recognized (Jiang et al. 2011). However, to the best of our knowledge, only one published study addressed the use of PBPK modeling to aid the development of alcoholresistant ER multi-particulate dosage form (Schrank et al. 2016).

The objectives of this study were: (i) to investigate biorelevance of different in vitro dissolution setups to assess ADD, and (ii) to evaluate the applicability of the conjoint in vitro-in silico approach in ADD-induced clinical risk assessment, using hydrophilic and lipophilic matrix tablets containing ibuprofen (IBU) and diclofenac sodium (DS) as model formulations. The selected model drugs are lipophilic compounds with poor aqueous solubility, therefore susceptible to increase in solubility, and possibly bioavailability (BA), in the presence of ethanol.

\section{Material and methods}

\section{Material and dosage forms}

Ibuprofen and diclofenac sodium were kindly donated by Galenika a.d., Serbia. All chemicals for media preparation were of analytical grade, purchased from the following suppliers: potassium dihydrogen phosphate, sodium hydroxide, and sodium chloride (Sigma-Aldrich, Germany), sodium dihydrogen phosphate (Centrohem d.o.o., Serbia), acetic acid glacial 99.5\% (Avantor Performance Materials-Macron Fine Chemicals, USA), hydrochlorid acid 37\%, and ethanol 96\% (v/v) (Zorka Pharma Hemija d.o.o., Serbia). Hydro-ethanolic media were prepared by replacing appropriate volumes of 
aqueous media with $96 \%$ (v/v) ethanol. The investigated dosage forms included commercially available IBU and DS SR tablets (Table 1).

[t]Table 1 near here[/t]

\section{Solubility determination}

Equilibrium solubility of the model drugs was tested in different media, including simple buffer solutions (0.1 M HCl pH 1.1, USP buffer solutions $\mathrm{pH} 6.8$ and $\mathrm{pH} 7.4$ ), and biorelevant media without surfactants (blank Fasted State Simulated Gastric Fluid (bFaSSGF) pH 1.6 (Vertzoni et al. 2005), blank Fasted State Simulated Intestinal Fluid (bFaSSIF) pH 6.5 (Galia et al. 1998) and Simulated Colonic Fluid (SCoF) pH 5.8 (Fotaki et al. 2005)) without/with the addition of $40 \%$ (v/v) ethanol. Excess amounts of model drugs were placed in a screw-cap vials containing $10 \mathrm{ml}$ of each tested media, and shaken at $75 \mathrm{rpm}$ in a shaking water bath (LSB Aqua Pro, Grant Instruments, UK) at $37^{\circ} \mathrm{C}$ for $24 \mathrm{~h}$. All experiments were run in triplicate. Samples were filtered through a $0.45 \mu \mathrm{m}$ MFTM membrane filter (Merck Millipore Ltd., IRL), properly diluted, and assayed UV-spectrophotometrically (Evolution 300, Thermo Fisher Scientific, UK) since this method has been referenced in literature for quantification of IBU and DS (Saei et al. 2008; Mourao et al. 2010; Rivera Leyva et al. 2012; Gonzales Vidal \& Gil Alegre 2013; USP 2016a; USP 2016b). Absorbance values were recorded at the wavelength of the relative maximum absorption for each drug (220-224 $\mathrm{nm}$ for ibuprofen, and 275-280 $\mathrm{nm}$ for diclofenac sodium, depending on the tested medium), and drugs concentrations were calculated from the relevant calibration curves.

\section{Dissolution study}

Drugs dissolution from the investigated SR tablets was tested under different experimental conditions: 
- (setup A) paddle apparatus (Erweka DT 70, Germany) at $50 \mathrm{rpm}$, using $900 \mathrm{ml}$ of a single medium $\mathrm{pH} 7.2$ (USP recommendation for ibuprofen immediate release (IR) tablets (USP 2016a)) or pH 7.5 (USP test 1 for diclofenac sodium ER tablets (USP 2016b)) without/with addition of $40 \%$ (v/v) ethanol;

- (setup B) basket apparatus (Erweka DT 70, Germany) at $100 \mathrm{rpm}$, using media change method: $900 \mathrm{ml} 0.1 \mathrm{M} \mathrm{HCl} \mathrm{pH} 1.1$ without/with addition of $40 \%$ (v/v) ethanol for $2 \mathrm{~h}, 900 \mathrm{ml} \mathrm{pH} 6.8$ for $2 \mathrm{~h}$, and $900 \mathrm{ml} \mathrm{pH} 7.4$ for $4 \mathrm{~h}$;

- (setup C) reciprocating cylinder apparatus (BioDis, Varian Inc., USA) operating at $20 \mathrm{dpm}$, using media change method with a vessel $(240 \mathrm{ml})$ change after 15 min (in case of non-ethanolic acidic medium) and then at $1 \mathrm{~h}$ time intervals, in total: $15 \mathrm{~min}$ bFaSSGF, $3 \mathrm{~h}$ bFaSSIF and $5 \mathrm{~h} \mathrm{SCoF}$ to simulate fasted state condition sans alcohol; or $2 \mathrm{~h}$ bFaSSGF with $40 \%$ (v/v) ethanol, $1 \mathrm{~h}$ bFaSSIF with $20 \%$ (v/v) ethanol, $2 \mathrm{~h}$ bFaSSIF and $3 \mathrm{~h}$ SCoF to simulate the 'worst case' scenario following alcohol intake (scenario C1). In addition, IBU products were tested under the scenario where ethanol is added solely in the simulated stomach fluid (scenario C2): $2 \mathrm{~h}$ bFaSSGF with $40 \%$ (v/v) ethanol, $3 \mathrm{~h}$ bFaSSIF and $3 \mathrm{~h}$ SCoF.

The first experimental setup (setup A) was based on the EMA recommendation on ADD assessment to use compendial dissolution medium proposed for routine testing (EMA 2016). However, due to the lack of regulatory recommendations for dissolution testing of IBU SR tablets, the selected medium - phosphate buffer $\mathrm{pH} 7.2$ conformed to the USP recommendation for IBU IR tablets. Ethanol content was set to $40 \%$ (v/v) to simulate the scenario when a dosage form is exposed to a strong alcohol beverage (Lennernäs 2009). 
The second set of conditions (setup B) followed the FDA requirements (Anand et al. 2011), with an adjustment that included media change after $2 \mathrm{~h}$ of testing in acidic milieu.

Setup C was based on the previously demonstrated 'bioperformance' in vitro test for IBU SR tablets under normal fasting (sans alcohol) conditions (Beloica et al. 2015). To investigate the influence of ethanol on drug release profiles, hypothetical scenarios that simulate the presence of ethanol solely in the stomach fluid or subsequent ethanol emptying to the small intestine (represented with dosage form transfer to bFaSSIF with half of the concentration of ethanol in acid) were assumed. In general, media change experimental setups were designed to simulate changes in physiological conditions as the drug travels along the gastrointestinal (GI) tract, and to approximate conditions in the stomach and proximal intestine, since ethanol is mostly absorbed through the gastric (10-20\%) and small intestine (80-90\%) mucosa (Levitt et al. 1997).

All experiments were run in triplicate, at $37 \pm 0.5^{\circ} \mathrm{C}$. After each sampling time, aliquots withdrawn for analysis were replaced by the equal volume of fresh medium. Withdrawn samples were filtered, and after appropriate dilution, assayed for drug concentration UV-spectrophotometrically at the wavelength of the relative maximum absorbance.

The obtained dissolution profiles were compared using similarity factor $\left(\mathrm{f}_{2}\right)$, where an $f_{2}$ value higher than 50 indicated that two dissolution profiles were statistically similar (FDA 2015).

\section{In silico absorption modeling}

Simcyp ${ }^{\circledR}$ Population-Based Simulator (v. 14.1; Certara $^{\text {TM }}$, USA) was used to simulate oral absorption of the model drugs, and estimate the influence of dissolution rate on drug bioavailability. Simulations were performed using the Advanced dissolution, 
absorption and metabolism (ADAM) model, that includes nine anatomically defined GI compartments (stomach, seven segments of the small intestine and colon), and describes drug absorption from each compartment as a function of release from a dosage form, dissolution, precipitation, luminal degradation, permeability, metabolism, transport, and transit through successive compartments. A detailed description of the model is described elsewhere (Jamei et al. 2009; Kostewicz et al. 2014). ADAM model was linked with full physiologically-based pharmacokinetic (PBPK) perfusion-limited model used to simulate drug distribution through different body tissues. The selected PBPK model provides a detailed description of 15 body compartments, including arterial and venous blood compartments, and uses a set of differential equations to describe physiological mechanisms that drive drug distribution in vivo (Jamei et al. 2014). The model comprises two sets of input parameters, namely system-related physiological parameters, and drug and formulation specific inputs (along with dosing regimen data). Physiological parameters were left at model default values for population representative subject of the Sim-Healthy Volunteers population in the fasted state. In cases when in vitro testing in acidic medium was performed for $2 \mathrm{~h}$, the simulated gastric emptying time was adjusted accordingly.

The required parameters related to the investigated drugs/dosage forms biopharmaceutical properties were taken from literature and/or in silico estimated, except for dissolution data, which were experimentally determined. Summary of drug specific inputs is given in Table 2. In vitro obtained dissolution profiles were used as an input function in ADAM to estimate the expected drug product in vivo performance. The tabulated in vitro dissolution data were entered under the 'IR dosage form' option, which better describes dissolution rate of poorly soluble drugs than Simcyp ${ }^{\circledR}$ 'Controlled/Modified Release' entry (Beloica et al. 2015). 
[t]Table 2 near here[/t]

The predictive power of the generated models was assessed by comparing the simulation results with the published data from bioavailabilty studies for IBU (Pargal et al. 1996) and DS (Rao et al. 2001) SR tablets. The relevant percent prediction error (\% $\mathrm{PE}$ ) for the pharmacokinetic parameters $\mathrm{C}_{\max }, \mathrm{t}_{\max }$ and $\mathrm{AUC}_{0-\mathrm{t}}$ were calculated using the following equation:

$$
\% \mathrm{PE}=\frac{(\text { Observed } \quad-\text { Pr edicted }) \times 100}{\text { Observed }}
$$

\section{Results}

\section{Solubility determination}

According to the Biopharmaceutics Classification System (BCS) criteria, both ibuprofen and diclofenac sodium belong to BCS class 2 drugs (Potthast et al. 2005;

Chuasuwan et al. 2009), meaning they are characterized by high intestinal permeability and low or absorption-limiting solubility. In addition, weakly acidic nature of the tested drugs (pKa 4.5 and 4.2 for IBU and DS, respectively) indicate they are expected to exhibit $\mathrm{pH}$-dependent solubility in the GI pH range. Experimentally obtained solubility values for the two drugs in different aqueous and hydro-ethanolic media are provided in Table 3.

The observed increase in IBU solubility with the increase of media $\mathrm{pH}$ was in line with the values reported in literature (Potthast et al. 2005). Solubility of DS also increased with the increase of media $\mathrm{pH}$; however, experimental values were higher than those reported previously (Chuasuwan et al. 2009). This deviation might be explained by the differences in the experimental conditions, i.e. these experiments were 
performed at $37^{\circ} \mathrm{C}$, while the published data relates to studies conducted at room temperature.

The addition of ethanol showed marked effect on the model drugs solubility, although it was dependent on the employed media composition. The highest increase in solubility in the presence of ethanol was observed for both drugs in acidic media $\mathrm{pH} 1.1$ and bFaSSGF pH 1.6 (Table 3). This is an important observation regarding that orally ingested alcohol mainly exhibit its solubilization effect in acidic stomach environment. According to the calculated dose-solubility (D/S) ratio (Table 3), under normal fasting conditions (without ethanol) in the stomach, model drugs are expected to show solubility-limited dissolution from the investigated $800 \mathrm{mg}$ IBU and $100 \mathrm{mg}$ DS tablets $(\mathrm{D} / \mathrm{S}>250 \mathrm{ml}$ ). This assumption is based on $240-250 \mathrm{ml}$ available gastric liquid upon the ingestion of a glass of water (240 ml) on an empty stomach (Mudie et al. 2014). But, due to increased drug solubility in the presence of $40 \%$ ethanol, concomitant intake of strong alcoholic beverages, especially in large volumes, might induce dose dumping from both IBU and DS SR tablets even at low $\mathrm{pH}$ in the stomach.

[t]Table 3 near here[/t]

Another annotation in this study concerns the observed drug-specific response to ethanol effect on drug solubility depending on buffer composition. According to the theory of Higuchi et al. (1953), diverse effect of buffer components, namely buffer ionic strength on IBU and DS solubilities in hydro-ethanolic media can be explained by differences in the polarity of the investigated drugs (i.e. DS is more polar than IBU and thus exhibit increased solubility with the increase of ionic strength of hydro-ethanolic media). Higher extent of ethanol-induced solubility increase at lower $\mathrm{pH}$ values also coincides with the finding of Higuchi et al. (1953). 


\section{Dissolution study}

\section{Ibuprofen}

In order to in vitro assess IBU dissolution from the investigated SR tablets without and in the presence of ethanol, different experimental setups have been employed. The results obtained under the setup A are depicted in Figure 1a. In the case of IBU1 hydrophilic matrix tablets drug release was slow and incomplete (less than $15 \%$ of drug released in $8 \mathrm{~h}$ ) irrespective of the addition of ethanol. Such results could be ascribed to the slow hydration of xanthan gum used as a matrix component. On the other hand, IBU2 lipophilic matrix tablets demonstrated abrupt drug release, which was further enhanced in the presence of ethanol. Visual inspection revealed that the main mechanism of drug release from IBU2 tablets is matrix erosion; therefore, it can be assumed that the accelerated drug dissolution at least partially resulted from increased solubility of ibuprofen in ethanolic solutions. Following the regulatory decision pathway for ADD risk assessment (EMA 2014; EMA 2016), the results obtained in medium $\mathrm{pH} 7.2$ indicate that IBU2 is an ethanol-vulnerable formulation, in contrast to IBU1. But, biorelevance of these data need to be taken with caution. Namely, different drug release profiles in the buffer medium without ethanol $\left(f_{2}=18.45\right)$ for the two products with proven bioequivalence (BE) in vivo (under fasting conditions without alcohol intake) clearly indicate that the employed dissolution method is over discriminatory. Therefore, dissolution results obtained in the presence of ethanol under the setup A might also be regarded as over discriminatory.

According to the results obtained under the setup B (Figure 1b), IBU dissolution during the first 2 hours was increased in hydro-ethanolic medium, with the effect being more evident in the case of IBU2 product. Subsequent change (increase) in media $\mathrm{pH}$ resulted in the increased drug dissolution from IBU2 formulation, while drug release 
from IBU1 was less affected. Xanthan gum, a component of IBU1, is practically insoluble in ethanol (Rowe et al. 2009); thus hydro-ethanolic medium is expected to have no or even some negative effect on the polymer swelling, and drug release rate from IBU1 (demonstrated by almost parallel drug release profiles between 2 and $8 \mathrm{~h}$ time points, Figure 1b). Initial increase in drug release from IBU1 in acidic medium with the addition of ethanol could be ascribed to the notably enhanced drug solubility in this medium (Table 3). The exact mechanism of the influence of ethanol on drug release from IBU2 is unknown, but it is evident that the addition of high percent ethanol interfere with drug release pattern. According to the strict FDA requirements (similar dissolution profiles during $2 \mathrm{~h}$ testing in $0.1 \mathrm{M} \mathrm{HCl}$ without and with ethanol), both IBU1 and, in a manner, IBU2 refer to rugged formulations $\left(\mathrm{f}_{2}=55.47\right.$ and $\mathrm{f}_{2} \approx 50$ (precisely 49.15) for IBU1 and IBU2, respectively). Comparison of the 8 h-profiles indicate ethanol-vulnerability for IBU2 tablets $\left(f_{2}=46.30\right)$. Again, there is a question of biorelevance of the results obtained under the described 'simple buffers media change' conditions, because the results obtained in aqueous media without ethanol signified erroneous differences $\left(f_{2}=43.08\right)$ between drug dissolution rates from the two bioequivalent products.

IBU dissolution was also tested in a reciprocating cylinder apparatus (setup C). As illustrated in Figure 1c, the two IBU products exhibited superimposed dissolution profiles under the media change setup without ethanol $\left(f_{2}=83.34\right)$. The influence of ethanol on drug release profiles is depicted in the profiles obtained under the conditions described as scenarios C2 and C1. The obtained profiles (Figure 1c), and the calculated similarity factors $\left(\mathrm{f}_{2}=71.61\right.$ and $\mathrm{f}_{2}=72.76$ for IBU1 in scenarios $\mathrm{C} 2$ and $\mathrm{C} 1$, respectively) indicate that IBU1 is an ADD-insensitive formulation. On the other hand, drug release from IBU2 tablets was significantly increased in hydro-ethanolic media $\left(\mathrm{f}_{2}\right.$ 
$=30.83$ and $\mathrm{f}_{2}=19.90$ for IBU2 in scenarios $\mathrm{C} 2$ and $\mathrm{C} 1$, respectively), specially under the scenario $\mathrm{C} 1$ conditions when ethanol was also present in the simulated small intestine medium (Figure 1c). In other words, the results suggest that IBU2 might exhibit ADD in vivo.

\section{Diclofenac sodium}

Dissolution testing of DS1 and DS2 SR tablets followed the experimental plan applied for IBU tablets, and the results are presented in Figure 2. Under the conditions proposed by the USP (phosphate buffer $\mathrm{pH} 7.5$ in paddle apparatus, setup A) the two products exhibited similar dissolution profiles $\left(f_{2}=70.61\right)$, showing gradual release of the drug (Figure $2 \mathrm{a}$ ). The addition of $40 \%$ ethanol had no effect on drug dissolution from DS1 ( $\mathrm{f}_{2}$ $=72.53)$; however it did affect drug release from DS2 tablets $\left(f_{2}=18.22\right)$. Abrupt drug release observed in the case of DS2 could in part be explained by increased solubility of cetyl alcohol, a component of tablet core, in hydro-ethanolic medium (Rowe et al. 2009), and high solubility of the released diclofenac at higher $\mathrm{pH}$ values. On the other hand, hypromellose-based DS1 retained the ability to control drug release in the presence of high ethanol concentration.

The employment of media change method in basket apparatus (setup B) gave different outcome, as illustrated on Figure $2 \mathrm{~b}$. Besides that there were no significant differences in dissolution behaviour between the two products $\left(f_{2}=72.24\right.$ in a setup without ethanol), the presence of ethanol in acidic medium showed no effect on drug release rate (similar dissolution profiles, data not shown). The obtained results reflect controlled drug release from both matrices under the selected conditions, with staircaseshaped profiles that followed the increase in DS solubility with increase in media $\mathrm{pH}$. It is interesting to note that under the employed test conditions both formulations showed ethanol-resistance in acidic medium (less than $10 \%$ of drug dissolved in $2 \mathrm{~h}$ ). 
The third set of data (Figure 2c) illustrates the results obtained under the conditions that correspond to the setup C. Drug dissolution in the acidic medium without ethanol was negligible, but subsequent increase in media $\mathrm{pH}$ shaped different drug release from DS1 and DS2 tablets. In the case of DS1 drug dissolution was gradual, whereas DS2 demonstrated more abrupt drug release (complete dissolution in less than $3 \mathrm{~h}$ ). It should be noted that in the absence of in vivo BE data for DS1 and DS2 tablets, there is not enough information to conclude whether the designed setup C provides biorelevant and discriminatory test conditions for the investigated DS tablets. However, published data on drug plasma concentration profile following oral administration of DS2 tablets (Popović et al. 2009) signify considerably higher $\mathrm{C}_{\max }$ $(3.86 \mu \mathrm{g} / \mathrm{ml})$ and AUC $(11.60 \mu \mathrm{g} \mathrm{h} / \mathrm{ml})$ in comparison to majority of data obtained in other BA/BE studies for $100 \mathrm{mg}$ SR DS tablets (average $\mathrm{C}_{\max } 1.04 \mu \mathrm{g} / \mathrm{ml}$ and AUC 3.60 $\mu \mathrm{g} \mathrm{h} / \mathrm{ml}$ for the data reported in (Davies \& Anderson 1997)), supporting the assumption that DS2 might indeed exert relatively fast dissolution in vivo. Exposure of the two DS products to hydro-ethanolic media that emulate the 'worst case' scenario (correspond to scenario $\mathrm{C} 1$ in the case of IBU tablets) had opposite effects on drug release rates. The obtained profile for DS1 suggests that addition of ethanol did not affect the integrity of hypromellose-based matrix, and initially decreased drug dissolution presumably resulted from prolonged $(2 \mathrm{~h})$ exposure of acidic drug to low $\mathrm{pH}$ medium. In contrast, DS2 exhibited rather fast matrix disruption in the presence of ethanol (visually observed tablet disintegration), which resulted in burst drug release.

\section{Drug-specific PBPK models}

In silico simulation of IBU absorption following administration of $800 \mathrm{mg}$ SR tablets was performed using previously developed and validated IBU-specific PBPK model (Beloica et al. 2015). The generated drug plasma concentration-time profiles, based on 
dissolution data obtained in the setup $\mathrm{C}$ without ethanol, are presented in Figure 3, together with the mean plasma profile observed in vivo (Pargal et al. 1996). Predicted and in vivo observed pharmacokinetic parameters are given in Table 4. The calculated PEs for AUC for both products were less than $10 \%$ in comparison to the mean data observed in the study of Pargal et al. (1996), indicating that the in vitro data from the proposed setup served as a good predictor of IBU oral absorption from SR tablets. The presented data also illustrate certain divergence between the predicted peak plasma concentrations for IBU1 and IBU2, which may arise from variable time of partial tablet disruption, as annotated in our previous study (Beloica et al. 2015).

Diclofenac-specific absorption model was generated using the selected input data provided in Table 2. In order to depict drug pharmacokinetic behaviour of DS SR tablets, dissolution data obtained under the setup $\mathrm{C}$ were selected as additional inputs to define drug release in vivo. Although the selected in vitro data indicated notable differences in the rate of drug release from the two formulations, several arguments supported the assumption that this scenario might actually happen in vivo. Namely, the results from BE study with DS2 tablets (Popović et al. 2009) indicated considerably higher $\mathrm{C}_{\max }$ and AUC in comparison to the average values observed for $100 \mathrm{mg}$ DS SR tablets (Davies \& Anderson 1997), and in addition several trials showed that pharmacokinetics of orally taken diclofenac are highly variable (Hooper et al. 1996; Lötsch et al. 2000; Garbacz et al. 2008).

The simulated profile for product DS1 coincided well with the average values observed in vivo (Figure 4, Table 4). Somewhat higher PE values for $\mathrm{t}_{\max }$ and AUC in comparison to the selected mean in vivo data (Table 4) can be regarded as reasonable estimates considering variable diclofenac pharmacokinetics. On the other hand, the simulated profile for DS2 diverged from the mean in vivo profile obtained in the study 
of Rao et al. (2001). The observed shift of $C_{\max }$ to higher values has indeed been noticed in some clinical trials with diclofenac SR tablets, including the already mentioned study that involved DS2 tablets (Suleiman et al. 1989; Popović et al. 2009). But, the simulated profile for DS2 with relatively sharp decrease in drug plasma concentration (Figure 4) indicate that drug therapeutic effect might not last as long as expected for SR tablet formulation.

[t]Table 4 near here[/t]

\section{In vitro-in silico approach in $A D D$ risk assessment}

In order to forecast IBU oral absorption in the presence of ethanol, two sets of dissolution data were used to describe the supposed course of drug release in vivo. The input dissolution profiles were obtained in the setup $\mathrm{C}$, under different conditions regarding the addition of ethanol (scenarios $\mathrm{C} 1$ and $\mathrm{C} 2$ ); the selected profiles are presented in Figure 1c. The generated pharmacokinetic data are shown in Figure 5 and Table 5. The simulated profiles for IBU1 revealed some reduction in $\mathrm{C}_{\max }$ in the presence of ethanol in comparison to the profile that represent drug intake with a glass of water, but there were no major fluctuations in AUC values (\% reduction less than 10\%) (Table 5). These findings support the idea that IBU1 is an alcohol-resistant formulation. As for IBU2, differences in drug release rate in vitro in the presence of ethanol were well reflected in the predicted pharmacokinetic profiles. Simulations revealed that $2 \mathrm{~h}$ exposure of IBU2 to $40 \%$ ethanol (scenario $\mathrm{C} 2$ ) is expected to increase peak drug plasma concentration for more than $40 \%$, and systemic bioavailability (expressed as AUC) for more than 20\% (Table 5). The in vivo impact of prolonged tablet exposure to ethanol, after drug and ethanol emptying into the small intestine (scenario $\mathrm{C} 1$ ), is anticipated to be even more pronounced. These results signify that 
IBU2 is prone to ADD in vivo. Still, such an increase in the extent of drug absorption is not expected to be of clinical significance since the simulated plasma concentrations did not cross therapeutic boundary (up to $50 \mu \mathrm{g} / \mathrm{ml}$ (Davies 1998)). Simulation based on the hypothetical dissolution scenario (profile V100, Figures 1c and 5, Table 5), demonstrated that even $100 \%$ drug release in the stomach would not lead to toxic drug concentrations in plasma (>100 $\mu \mathrm{g} / \mathrm{ml}$ (Davies 1998)).

[t]Table 5 near here[/t]

The influence of strong alcoholic beverages on diclofenac absorption from the SR tablets was assessed based on the 'worst case' in vitro dissolution scenario (scenario C1) results (shown in Figure 2c). According to the simulation outcomes (Figure 6), concomitant alcohol intake is expected to decrease DS1 peak plasma concentration in case of sufficiently long (e.g. 2 h) tablet residence time in the stomach (near 30\% reduction in $\mathrm{C}_{\max }$, Table 5). However, as discussed above, this outcome most likely arise from decreased drug solubility in acidic medium, and not ethanol effect on the hypromellose matrix performance. Therefore, it may be assumed that the effect of ethanol on drug absorption from DS1 will predominantly be expressed through the influence on gastric emptying time. The simulated data for DS2 also indicated marked decrease in $\mathrm{C}_{\max }$ when tablets were ingested with alcoholic drink (due to relatively fast elimination of the rapidly released drug (Davies 1998; Altman et al. 2015)), but the overall exposure (measured by AUC) was not significantly changed (Table 5). In addition, it should be noted that neither of the predicted scenarios regarding diclofenac absorption in the presence of ethanol are expected to pose safety issues for patients, since the simulated drug plasma concentrations were within the therapeutic range (Davies 1998). 


\section{Discussion}

This study describes a step-by-step approach to assess the risk of ADD for SR oral dosage forms, and elucidate the expected clinical outcomes of burst or altered drug release. The proposed approach was tested using two model drugs with $\mathrm{pH}$-dependent solubility, available as both hydrophilic and lipophilic SR tablets.

It is well known that ethanol-induced increase in drug solubility may lead to ADD, but the obtained results revealed that the magnitude of this effect depends upon characteristics of a particular drug and composition of hydro-ethanolic media. Another annotation was that ethanol exhibited the largest effect on acidic drug solubility at low $\mathrm{pH}$ corresponding to the fasting stomach environment. However, the overall in vitro (solubility and dissolution) results suggested that the impact of ethanol on drug release from SR tablets was predominantly governed by the influence on matrix performance, and less by increase in drug solubility.

The second part of the study aimed to assess the suitability of different dissolution setups to forecast potential ADD for the investigated SR oral formulations. The obtained results clearly demonstrate that the decision on ethanol-vulnerability of a test formulation depends on the employed experimental conditions. This is well illustrated in the case of DS1 and DS2 products that appeared to be ethanol-resistant based on dissolution data obtained under the conditions that conform with the FDA recommendations $(2 \mathrm{~h}$ testing in $0.1 \mathrm{M} \mathrm{HCl})$. However, testing in a compendial medium (buffer $\mathrm{pH}$ 7.5), as proposed by EMA, was more discriminatory, and indicated significant differences in the rate of drug release form DS2 in the presence of ethanol (ethanol-vulnerable formulation). Testing of IBU tablets under different experimental conditions also resulted in different dissolution outcomes; however, each of the employed dissolution setups pointed out to the conclusion that IBU1 is ethanol- 
resistant, and IBU2 ethanol-vulnerable formulation (conditionally, based on the first $2 \mathrm{~h}$ dissolution in $0.1 \mathrm{M} \mathrm{HCl}$ IBU2 might also be treated as rugged). Regarding the diversity of the obtained results, the predictive power of different dissolution setups to demonstrate ADD should be critically assessed. According to the obtained results, neither FDA nor EMA proposed dissolution test conditions seemed suitable for the in vitro assessment of ADD in cases of IBU and DS tablets. This was demonstrated in the lack of biorelevance of the initial setup (setup A or B) that comprised media without ethanol, as illustrated in IBU example. Namely, under the proposed setups A and B (with no ethanol in media), the two IBU products with proven bioequivalence under fasting conditions in vivo showed different dissolution profiles in vitro (Figures 1a and b).

Another annotation regarding biorelevance of the FDA proposed setup concerns the required $2 \mathrm{~h}$ testing in acidic medium without and with addition of ethanol. Two hours tablet residence in acidic medium without ethanol can only serve for direct comparison of the resultant dissolution profiles with the profile obtained in hydroethanolic medium, and/or for testing of formulation acid-resistance. Therefore, it may be suitable for routine quality control or in the early phases of formulation design related to alcohol-sensitivity testing. But regarding that such prolonged drug residence time is not expected under normal (sans ethanol) fasting conditions, the obtained in vitro data cannot be considered predictive of drug product in vivo behavior. For these reasons, we propose an alternative in vitro setup (testing in a reciprocating cylinder apparatus, using media change principle), suggested as bioperformance dissolution test for IBU SR tablets (Beloica et al. 2015). Still, there are no data available from in vivo ADD studies to justify whether the proposed in vitro setup adequately simulate ethanol effect on drug release. The selected conditions comprising $40 \%$ ethanol in acidic 
medium for $2 \mathrm{~h}$, followed by $1 \mathrm{~h}$ testing in simulated intestinal medium with $20 \%$ ethanol might be considered as 'too extreme', but may actually represent realistic scenario, depending on the volume, duration and content of the ingested alcohol beverage (Lennernäs 2009). In this context, additional experiments with different formulations and model drugs are needed to demonstrate if the proposed in vitro setup might be recommended as a universal solution for ADD risk assessment of SR oral products.

The results obtained in the setup $\mathrm{C}$ indicate that the investigated xanthan gum and hypromellose-based matrix SR tablets are rather insensitive to ADD, while lipophilic matrices investigated in this study are potentially alcohol-vulnerable and may exhibit dose dumping following concomitant alcohol intake. However, in vitro dissolution testing can be over discriminatory. For example, simulation results for DS2 (Table 5) revealed only small differences in AUC in the presence and absence of ethanol, although dissolution data indicated significant differences in drug release kinetics. This further implies that the employment of in vitro dissolution testing alone might not be able to provide answers on alcohol-vulnerability of a specific drug product in vivo. The major reason is that, apart from formulation factors, physiological processes also influence drug release in vivo, and the full complexity of this influence cannot be simulated in vitro. In this context, the combined in vitro-in silico approach may provide better insight into the effect of concomitant alcohol intake on drug clinical performance.

Simulation results also revealed that the observed changes in drug dissolution rate in the presence of ethanol are expected to affect drug absorption rate and change the shape of plasma concentration-time profile (Figures 5 and 6). However, none of the predicted scenarios for IBU and DS tablets indicated the risk of potentially dangerous 
ADD. Regarding this observation, it would be prudent to distinguish between alcoholinduced abrupt drug release and alcohol-induced changes in drug plasma exposure. The first term applies to the inability of the formulation to control drug release; this phenomenon can influence drug absorption profile, but not necessarily because drug plasma exposure also depends upon drug pharmacokinetic properties. On the other hand, alcohol-induced change in drug plasma exposure can be defined as significant, potentially dangerous increase/decrease in drug plasma concentration, and should be considered in regard to drug therapeutic range and the expected therapeutic effect. Alcohol-induced changes in drug plasma exposure may pose a serious risk for patients either due to safety issues or diminished efficacy or both. These two phenomena are generally linked, but depending on the drug pharmacokinetic properties and interindividual variations in GI physiology, occurence of one of them does not necessarily mean that the other one will also happen. Therefore, it is equally important to investigate drug dissolution in the presence of ethanol in vitro, and to forecast the expected pharmacokinetic outcome using in silico tools.

Additionally, our study with SR products containing nonsteroidal antiinflammatory drugs (NSAIDs) supports the endeavours to expand ADD risk assessment studies to all SR oral dosage forms, not just to those containing opioid analgetics. Although burst drug release in case of NSAIDs might not pose safety risk for patients (due to relatively high therapeutic index), potential influence of ethanol on drug solubility and/or tablet matrix integrity may cause the product to lose its modified release characteristics, and the expected duration of therapeutic effect.

Based on the strategy applied in this study, we may propose the following approach for ADD risk assessment: (i) construction of a drug-specific PBPK model, (ii) in vitro dissolution testing under biorelevant conditions 'sans alcohol' (predictability of 
the in vitro test can be confirmed by comparison with the results from clinical $\mathrm{BA} / \mathrm{BE}$ studies using either IVIVC or mechanistic PBPK modeling), (iii) in vitro dissolution testing under 'adjusted' experimental conditions to simulate the 'worst case' scenario that might be expected after alcohol intake (e.g. $2 \mathrm{~h}$ dissolution in acidic medium containing $40 \%$ ethanol, eventually followed by testing in simulated intestinal fluid with the addition of ethanol), (iv) in silico estimation of the expected plasma concentration-time profiles based on the input dissolution profiles obtained in vitro in the presence of ethanol.

\section{Conclusion}

The results of this study demonstrate that currently recommended methods for the in vitro assessment of ADD and ethanol-vulnerability of MR oral drug products are not necessarily predictive of drug in vivo behaviour following concomitant alcohol intake. In addition, an alternative dissolution setup that includes testing in a reciprocating cylinder apparatus, using media change has been suggested for biopredictive in vitro testing of potential ADD.

The presented data also demonstrate superiority of the combined in vitro-in silico approach over simple in vitro testing for ADD risk assessment, since the proposed approach may provide insight into the effect of ADD on drug plasma exposure. This approach should be encouraged, and used to complement formulation development of MR oral dosage forms.

\section{Acknowledgments}

This work was supported by the Ministry of Education, Science and Technological Development, Republic of Serbia [project TR34007]. 


\section{Disclosure of interest}

The authors report no declarations of interest.

\section{References}

Altman R, Bosch B, Brune K, Patrignani P, Young C. 2015. Advances in NSAID development: Evolution of diclofenac products using pharmaceutical technology. Drugs. 75:859-877.

Anand O, Yu L, Conner D, Davit B. 2011. Dissolution testing for generic drugs: an FDA perspective. AAPS J. 13:328-335.

Beloica S, Cvijić S, Bogataj M, Parojčić J. 2015. In vitro-in vivo-in silico approach in biopharmaceutical characterization of ibuprofen IR and SR tablets. Eur J Pharm Sci. 75:151-159.

Brennan PL, Schutte KK, Moos RH. 2005. Pain and use of alcohol to manage pain: Prevalence and 3-year outcomes among older problem and non-problem drinkers. Addiction. 100:777-786.

Chuasuwan B, Binjesoh V, Polli JE, Zhang H, Amidon GL, Junginger HE, Midha KK, Shah VP, Stavchansky S, Dressman JB, Barends DM. 2009. Biowaiver monographs for immediate release solid oral dosage forms: Diclofenac sodium and diclofenac potassium. J Pharm Sci. 98: 1206-1219.

Davies NM. 1998. Clinical Pharmacokinetics of Ibuprofen The First 30 Years. Clin Pharmacokinet. 34:101-154.

Davies NM, Anderson KE. 1997. Clinical Pharmacokinetics of Diclofenac Therapeutic Insights and Pitfalls. Clin Pharmacokinet. 33:184-213.

[EMA] The European Medical Agency. 2014. Guideline on the pharmacokinetic and clinical evaluation of modified release dosage forms. London: Committee for Medicinal Products for Human Use (CHMP). (EMA/CPMP/EWP/280/96 Corr1).

EMA: Quality of medicines Questions and Answers: Part 2, Subchapter: Specific types of product - Need for in vitro dissolution studies with alcohol for modified release oral products including opioid drug products [Internet]. c1995-2017. London: European Medical Agency; [cited 2016 Nov 20]. Available from: http://www.ema.europa.eu/ema/index.jsp?curl=pages/regulation/q_and_a/q_and _a_detail_000072.jsp\#section10. 
Fadda HM, Mohamed MAM, Basit AW. 2008. Impairment of the in vitro drug release behaviour of oral modified release preparations in the presence of alcohol. Int $\mathrm{J}$ Pharm. 360:171-176.

FDA: FDA Alert [7/2005]: Alcohol-Palladone Interaction [Internet]. c2005-2017. Silver Spring (MD): Food and Drug Administration; [updated 2015 Jul 15; cited 2016 Dec 3]. Available from: http://www.fda.gov/Drugs/DrugSafety/PostmarketDrugSafetyInformationforPati entsandProviders/ucm129288.htm.

[FDA] The Food and Drug Administration. 2008. Draft Guidance on Trospium Chloride. Rockville (MD): Food and Drug Administration.

[FDA] The Food and Drug Administration. 2010. Draft Guidance on Clonidine. Rockville (MD): Food and Drug Administration.

[FDA] The Food and Drug Administration. 2011. Quality by Design for ANDAs: An Example for Modified Release Dosage Forms. Rockville (MD): Food and Drug Administration.

[FDA] The Food and Drug Administration. 2014a. Draft Guidance on Metoprolol Succinate. Rockville (MD): Food and Drug Administration.

[FDA] The Food and Drug Administration. 2014b. Draft Guidance for Industry: Bioavailability and Bioequivalence Studies Submitted in NDAs or INDs General Considerations. Rockville (MD): Food and Drug Administration, Center for Drug Evaluation and Research (CDER).

[FDA] The Food and Drug Administration. 2014c. Draft Guidance on Metformin Hydrochloride; Sitagliptin Phosphate. Rockville (MD): Food and Drug Administration.

[FDA] The Food and Drug Administration. 2014d. Draft Guidance on Memantine Hydrochloride. Rockville (MD): Food and Drug Administration.

[FDA] The Food and Drug Administration. 2015. Draft Guidance for Industry: Waiver of In vivo Bioavailability and Bioequivalence Studies for Immediate Release Solid Oral Dosage Forms based on a Biopharmaceutics Classification System. Rockville (MD): Food and Drug Administration, Center for Drug Evaluation and Research (CDER).

[FDA] The Food and Drug Administration. 2016a. Draft Guidance on Dexlansoprazole. Rockville (MD): Food and Drug Administration. 
FDA: Product-specific recommendations for generic drug development [Internet]. 2016b. Silver Spring (MD): Food and Drug Administration; [updated 2016 Dec 1; cited 2016 Dec 2]. Available from: http://www.fda.gov/Drugs/GuidanceComplianceRegulatoryInformation/Guidanc es/ucm075207.htm.

Fotaki N, Symillides M, Reppas C. 2005. In vitro versus canine data for predicting input profiles of isosorbide-5-mononitrate from oral extended release products on a confidence interval basis. Eur J Pharm Sci. 24:115-122.

Friebe T. 2015. Alcohol-induced dose dumping intentional/accidental - IPEC Working Group. Proceedings of the IPEC Europe Excipients Forum; 2015 Feb 5; Nice, France.

Friebe T, Asgarzadeh F, Gray A, Hughes K, Hebestreit JP, Rosiaux Y, Yunis M, Faham A. 2015. Regulatory considerations for alcohol-induced dose dumping of oral modified-release formulations. Pharm Technol. 38:40-46.

Galia E, Nicolaides E, Hörter D, Löbenberg R, Reppas C, Dressman JB. 1998. Evaluation of various dissolution media for predicting in vivo performance of class I and II drugs. Pharm Res. 15:698-705.

Garbacz G, Wedemeyer RS, Nagel S, Giessmann T, Mönnikes H, Wilson CG, Siegmund W, Weitschies W. 2008. Irregular absorption profiles observed from diclofenac extended release tablets can be predicted using a dissolution test apparatus that mimics in vivo physical stresses. Eur J Pharm Biopharm. 70:421428.

Gonzales Vidal NL, Gil Alegre ME. 2013. UV Analytical Method Suitability for Investigation of BCS Class 2 Biowaivers: Ibuprofen Case. Dissol Technol. $20: 44-47$.

Henderson LS, Tenero DM, Campanile AM, Baidoo CA, Danoff TM. 2007. Ethanol does not alter the pharmacokinetic profile of the controlled-release formulation of carvedilol. J Clin Pharmacol. 47:1358-1365.

Higuchi T, Gupta M, Busse LW. 1953. Influence of electrolytes, $\mathrm{pH}$, and alcohol concentration on the solubilities of acidic drugs. J Am Pharm Assoc. 42:157161.

Hooper IT, Allen E, Mclaughlin K, Ward C, Sioufi A. 1996. Bioavailability of a generic sustained-release formulation of diclofenac compared with the standard sustained-release formulation. Clin Drug Invest. 12:259-270. 
Jamei M, Bajot F, Neuhoff S, Barter Z, Yang J, Rostami-Hodjegan A, Rowland-Yeo K. 2014. A mechanistic framework for in vitro-in vivo extrapolation of liver membrane transporters: Prediction of drug-drug interaction between rosuvastatin and cyclosporine. Clin Pharmacokinet. 53:73-87.

Jamei M, Turner D, Yang J, Neuhoff S, Polak S, Rostami-Hodjegan A, Tucker G. 2009. Population-based mechanistic prediction of oral drug absorption. AAPS J. $11: 225-237$.

Jedinger N, Khinast J, Roblegg E. 2014. The design of controlled-release formulations resistant to alcohol-induced dose dumping - A review. Eur J Pharm Biopharm. 87:217-226.

Jiang W, Kim S, Zhang X, Lionberger RA, Davit BM, Conner DP, Yu LX. 2011. The role of predictive biopharmaceutical modeling and simulation in drug development and regulatory evaluation. Int J Pharm. 418:151-160.

Kasim NA, Whitehouse M, Ramachandran C, Bermejo M, Lennernäs H, Hussain AS, Junginger HE, Stavchansky SA, Midha KK, Shah VP, Amidon GL. 2004. Molecular properties of WHO essential drugs and provisional biopharmaceutical classification. Mol Pharm. 1:85-96.

Kostewicz ES, Abrahamsson B, Brewster M, Brouwers J, Butler J, Carlert S, Dickinson PA, Dressman J, Holm R, Klein S, et al. 2014. In vitro models for the prediction of in vivo performance of oral dosage forms. Eur J Pharm Sci. 57:342-366.

Kumar S, Koppara S, Subramanian R, Braun M, Stearns R, Chiu S, Evans D, Baillie T. 2002. Extrapolation of diclofenac clearance from in vitro microsomal metabolism data: role of acyl glucuronidation and sequential oxidative metabolism of the acyl glucuronide. J Pharmacol Exp Ther. 303:969-978.

Lennernäs H. 2009. Ethanol-drug absorption interaction: Potential for a significant effect on the plasma pharmacokinetics of ethanol vulnerable formulations. Mol Pharm. 6:1429-1440.

Levina M, Vuong H, Rajabi-Siahboomi AR. 2007. The influence of hydro-alcoholic media on hypromellose matrix systems. Drug Dev Ind Pharm. 33:1125-1134.

Levitt MD, Li R, Demaster EG, Elson M, Furne J, Levitt DG. 1997. Use of measurements of ethanol absorption from stomach and intestine to assess human ethanol metabolism. Am J Physiol. 273:G951-G957.

Lötsch J, Kettenmann B, Renner B, Drover D, Brune K, Geisslinger G, Kobal G. 2000. Population pharmacokinetics of fast release oral diclofenac in healthy 
volunteers: Relation to pharmacodynamics in an experimental pain model. Pharm Res. 17:77-84.

Meyer RJ, Hussain AS. 2005. Awareness topic: mitigating the risks of ethanol induced dose dumping from oral sustained/controlled release dosage forms. FDA's ACPS Meeting; 2005 Oct 26; Rockville (MD), US.

Mourao SC, Da Silva C, Bresolin TM, Serra CH, Porta V. 2010. Dissolution parameters for sodium diclofenac-containing hypromellose matrix tablet. Int J Pharm. 386:201-207.

Mudie DM, Murray K, Hoad CL, Pritchard SE, Garnett MC, Amidon GL, Gowland PA, Spiller RC, Amidon GE, Marciani L. 2014. Quantification of gastrointestinal liquid volumes and distribution following a $240 \mathrm{~mL}$ dose of water in the fasted state. Mol Pharm. 11:3039-3047.

Obach RS. 1999. Prediction of human clearance of twenty-nine drugs from hepatic microsomal intrinsic data: an examination of in vitro half-life approach and nonspecific binding to microsomes. Drug Metab Dispos. 27:1350-1359.

Pargal A, Kelkar MG, Nayak PJ. 1996. The effect of food on the bioavailability of ibuprofen and flurbiprofen from sustained release formulations. Biopharm Drug Dispos. 17:511-519.

Pobudkowska A, Domańska U. 2014. Study of pH-dependent drugs solubility in water. Chem Ind Chem Eng Q. 20:115-126.

Popović J, Mikov M, Sabo A, Jakovljević V. 2009. Evaluation of statistical power function for various diclofenac bioequivalence trials with different subject numbers. Eur J Drug Metab Pharmacokinet. 34:85-91.

Potthast H, Dressman JB, Junginger HE, Midha KK, Oeser H, Shah VP, Vogelpoel H, Barends DM. 2005. Biowaiver monographs for immediate release solid oral dosage forms: Ibuprofen. J Pharm Sci. 94:2121-2131.

Rao YM, Veni JK, Jayasagar G. 2001. Formulation and evaluation of diclofenac sodium using hydrophilic matrices. Drug Dev Ind Pharm. 27:759-766.

Rivera-Leyva JC, García-Flores M, Valladares-Mendez A, Orozco-Castellanos LM, Martínez-Alfaro M. 2012. Comparative Studies on the Dissolution Profiles of Oral Ibuprofen Suspension and Commercial Tablets using Biopharmaceutical Classification System Criteria. Indian J Pharm Sci. 74:312-318.

Rowe R, Sheskey P, Quinn M, editors. 2009. Handbook of Pharmaceutical Excipients, 6th edition. London: Pharmaceutical Press. 
Saei AA, Jabbaribar F, Fakhree MAA, Acree Jr. WE, Jouyban A. 2008. Solubility of sodium diclofenac in binary water + alcohol solvent mixtures at $25^{\circ} \mathrm{C}$. J Drug Del Sci Tech. 18:149-151.

Schrank S, Jedinger N, Wu S, Piller M, Roblegg E. 2016. Pore blocking: An innovative formulation strategy for the design of alcohol resistant multi-particulate dosage forms. Int J Pharm. 509:219-228.

Suleiman MS, Najib N, EI-Sayed Y, Hasan M, Abdulahameed M. 1989. A study on the relative bioavailability of a sustained-release formulation of diclofenac sodium. Int J Clin Pharmacol Ther Toxicol. 27:276-279.

Tang W, Stearns R, Kwei G, Iliff S, Miller R, Egan M, Yu N, Dean D, Kumar S, Shou $\mathrm{M}$, et al. 1999. Interaction of diclofenac and quinidine in monkeys: Stimulation of diclofenac metabolism. J Pharmacol Exp Ther. 291:1068-1074.

[USP] The United States Pharmacopeial Convention. 2016a. The United States Pharmacopeia and National Formulary (USP40-NF35): Ibuprofen tablets. Rockville (MD): U.S. Pharmacopeial Convention.

[USP] The United States Pharmacopeial Convention. 2016b. The United States Pharmacopeia and National Formulary (USP40-NF35): Diclofenac sodium extended-release tablets. Rockville (MD): U.S. Pharmacopeial Convention.

Vertzoni M, Dressman J, Butler J, Hempenstall J, Reppas C. 2005. Simulation of fasting gastric conditions and its importance for the in vivo dissolution of lipophilic compounds. Eur J Pharm Biopharm. 60:413-417.

Willis JV, Kendall MJ, Ainn RM, Thornhill DP, Welling PG. 1979. The pharmacokinetics of diclofenac sodium following intravenous and oral administration. Eur J Clin Pharmacol. 16:405-410.

[WHO] The World Health Organization. 2014. Global status report on alcohol and health-2014 ed. Geneva: World Health Organization; [cited 2016 Dec 3].

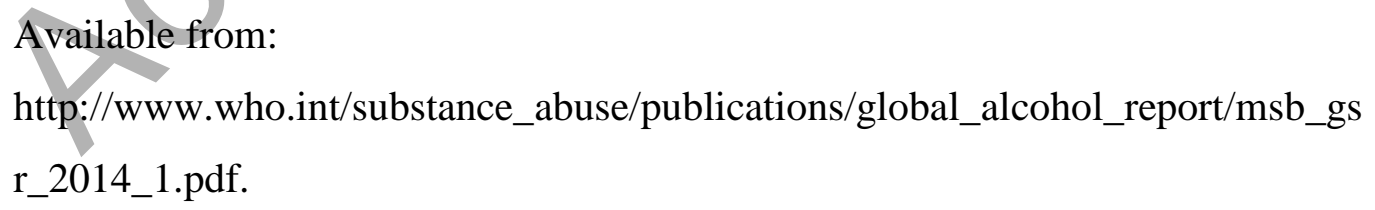

Yazdanian M, Briggs K, Jankovsky C, Hawi A. 2004. The "high solubility” definition of the current. FDA guidance on biopharmaceutical classification system may be too strict for acidic drugs. Pharm Res. 21:293-299. 
Figure 1. Ibuprofen SR tablets drug release profiles obtained under setup A (a), setup B (b), and setup C (c); closed circles refer to product IBU1, and open circles to product IBU2; V100 refer to the virtual dissolution profile illustrating $100 \%$ drug release in the stomach.

Figure 2. Diclofenac sodium SR tablets drug release profiles obtained under setup A (a), setup B (b), and setup C (c); closed circles refer to product DS1, and open circles to product DS2.

Figure 3. IBU plasma concentration-time profiles simulated on the basis of in vitro dissolution data obtained under the setup $\mathrm{C}$ without ethanol, along with the mean profile observed in vivo (Pargal et al. 1996).

Figure 4. DS plasma concentration-time profiles simulated on the basis of in vitro dissolution data obtained under the setup $\mathrm{C}$ without ethanol, along with the mean profile observed in vivo (Rao et al. 2001).

Figure 5. IBU1 (thick lines) and IBU2 (thin lines) plasma concentration-time profiles simulated based on dissolution data obtained under different conditions that comply with the setup C; V100 denotes the profile simulated based on virtual dissolution profile (100\% drug release in the stomach).

Figure 6. DS1 (thick lines) and DS2 (thin lines) plasma concentration-time profiles simulated based on dissolution data obtained under different conditions that comply with the setup C. 
(a)
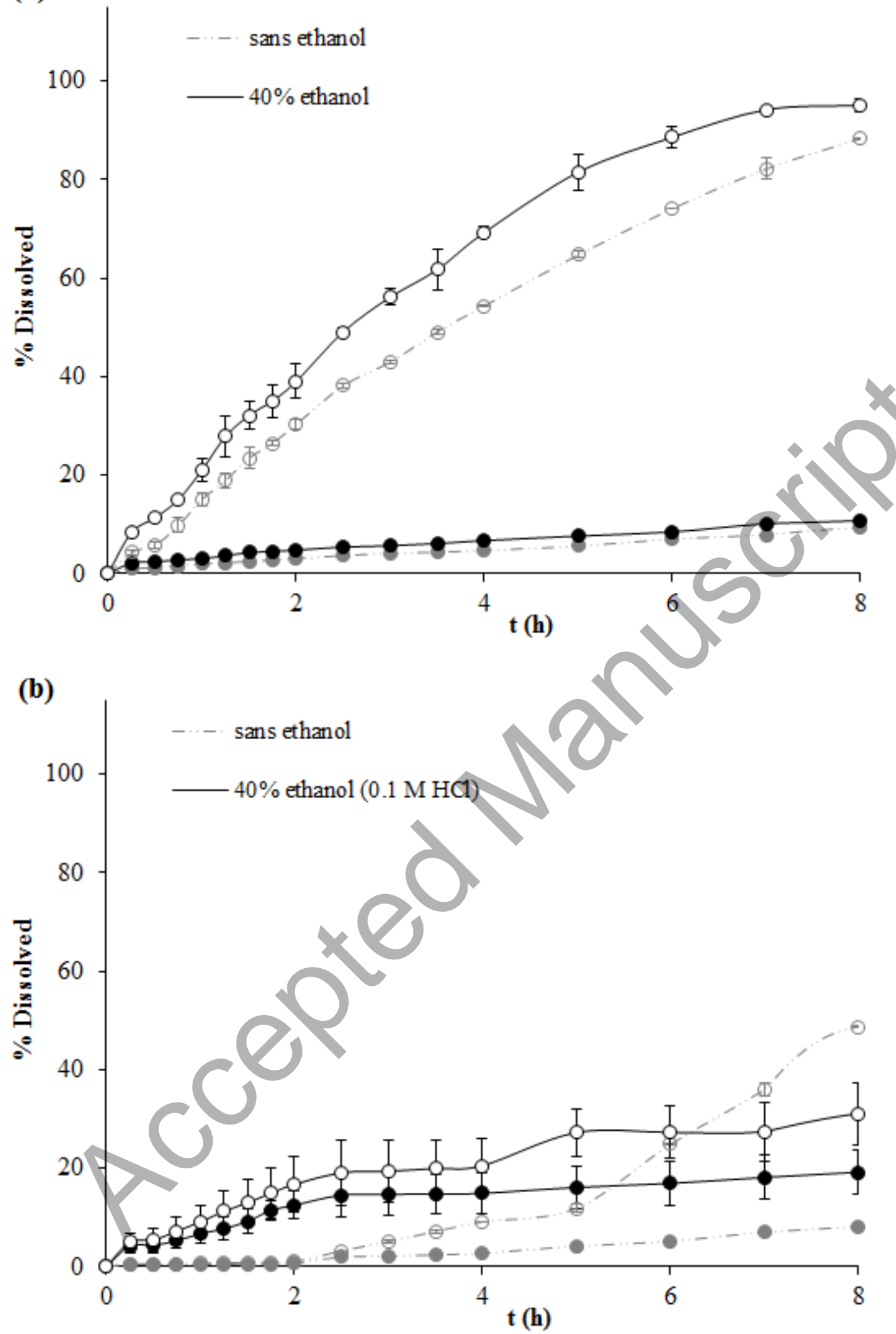
(c)

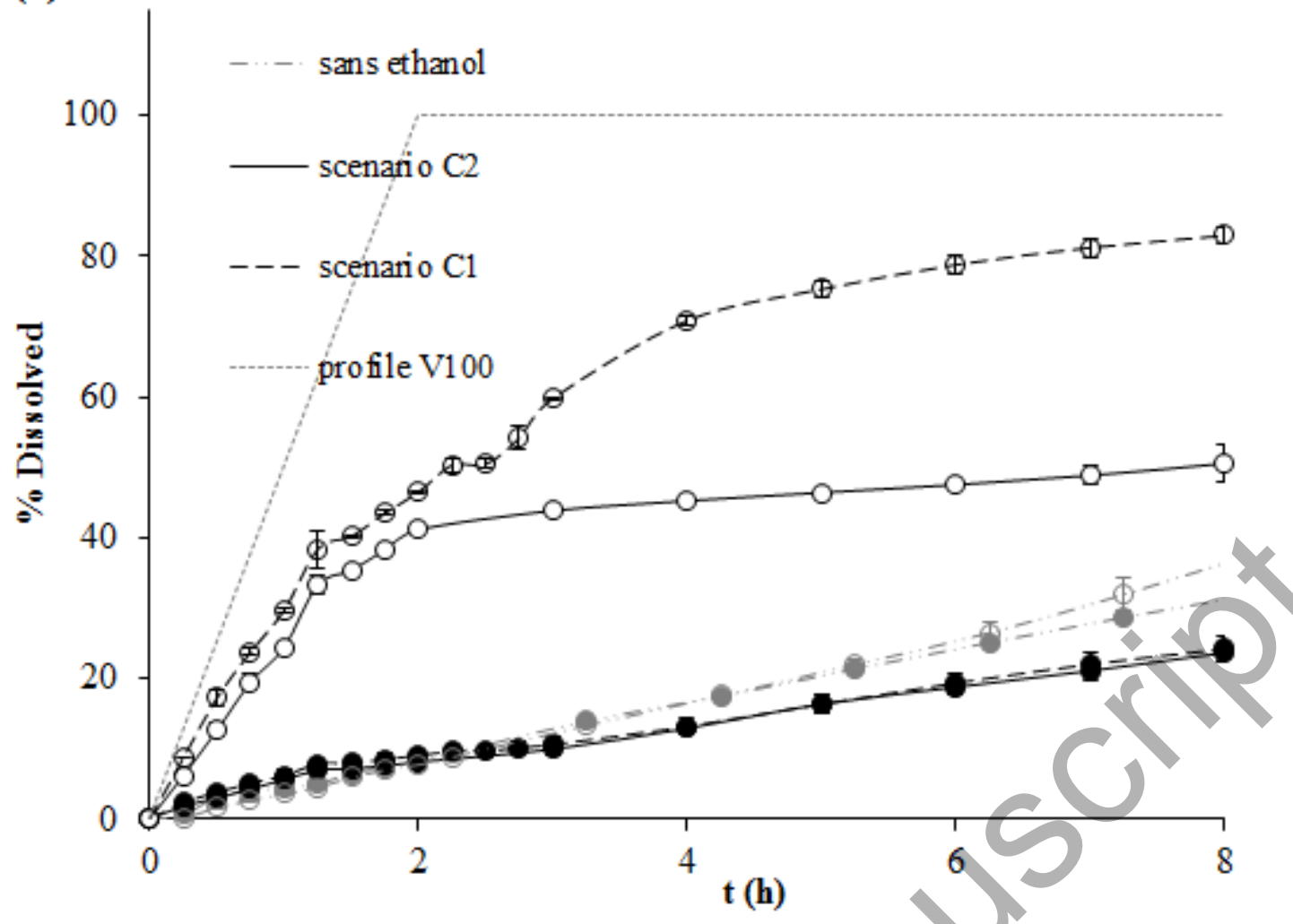

(a)

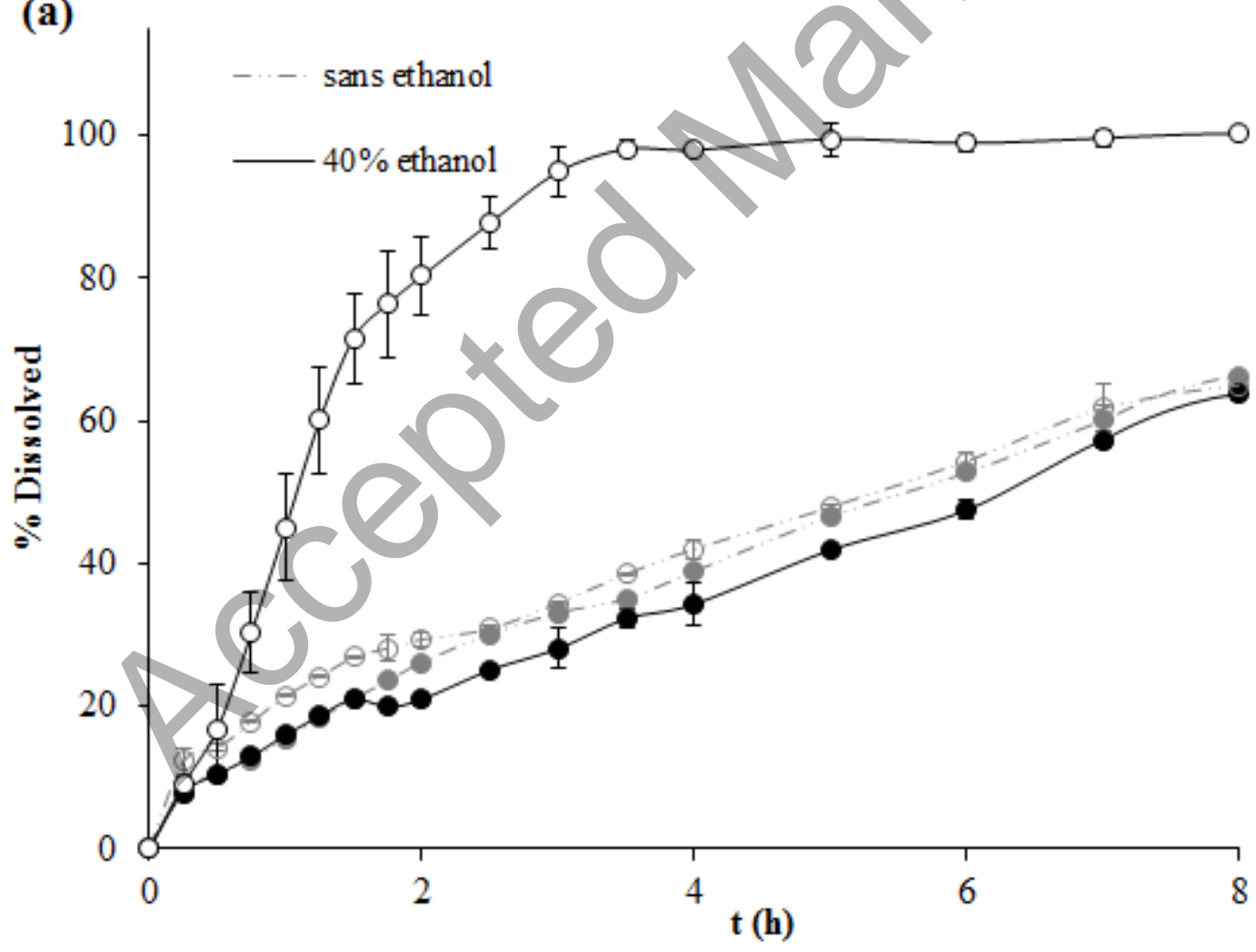



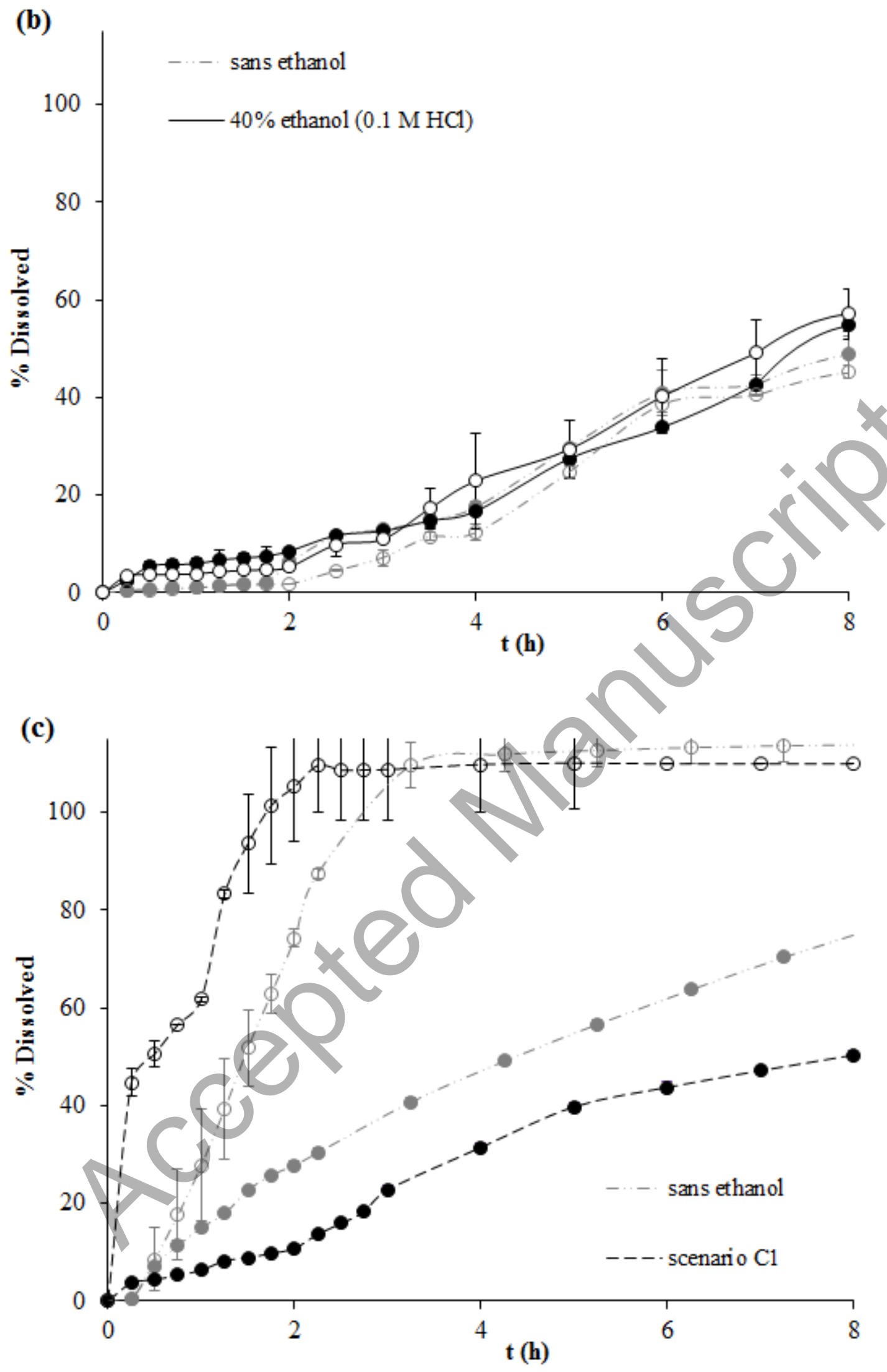

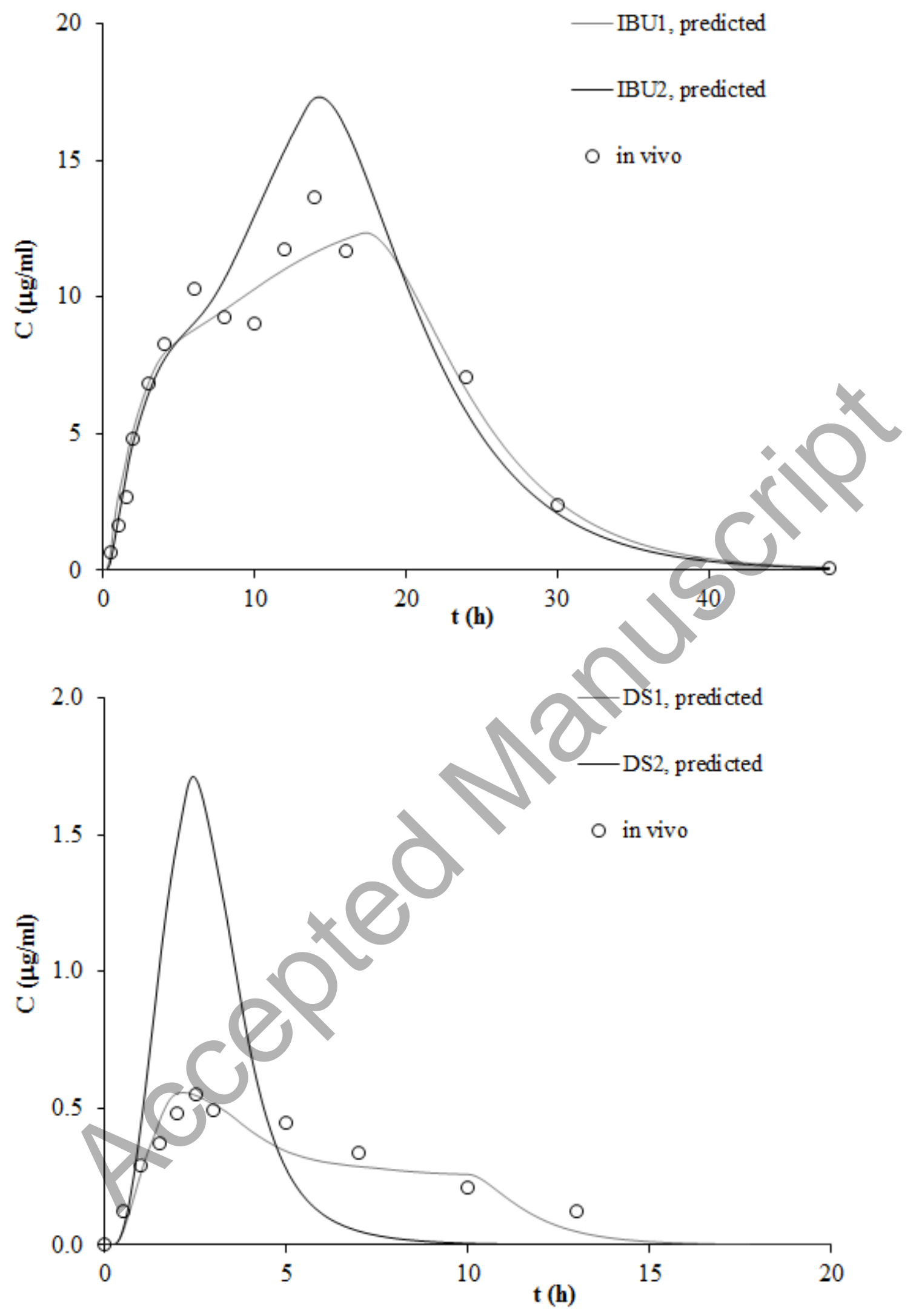

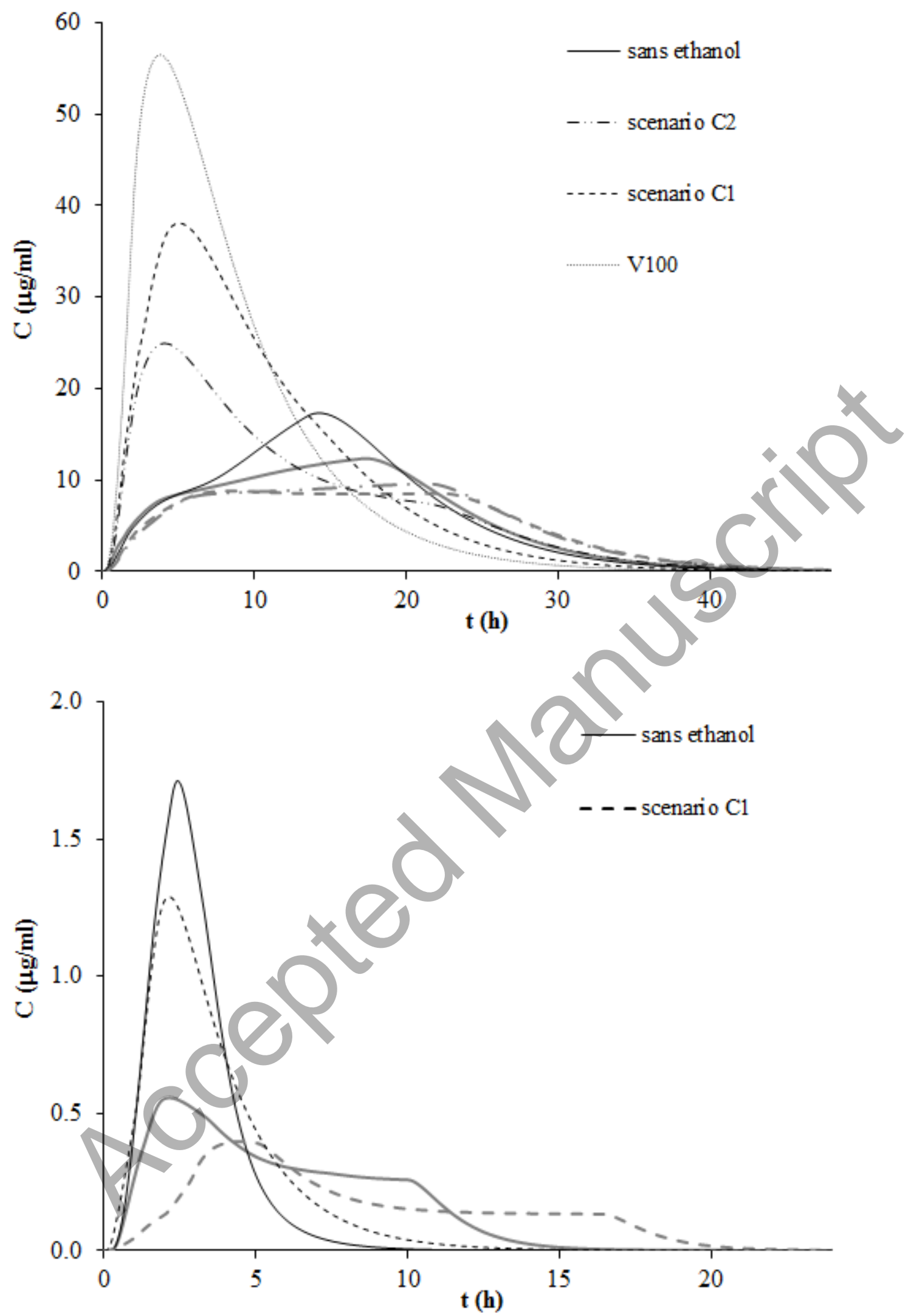
Table 1. Composition of the investigated SR tablets.

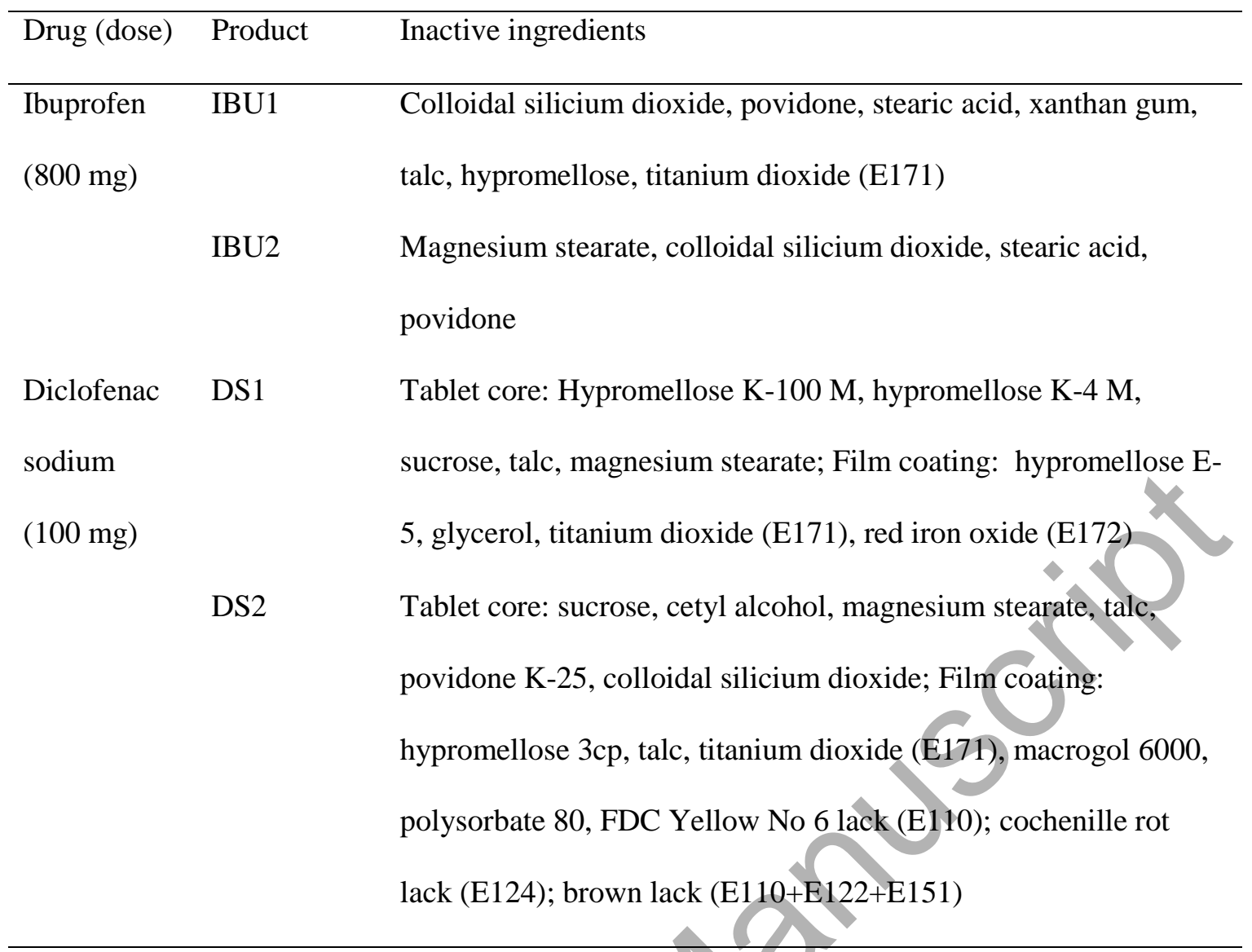


Table 2. Summary of input parameters employed for the simulations.

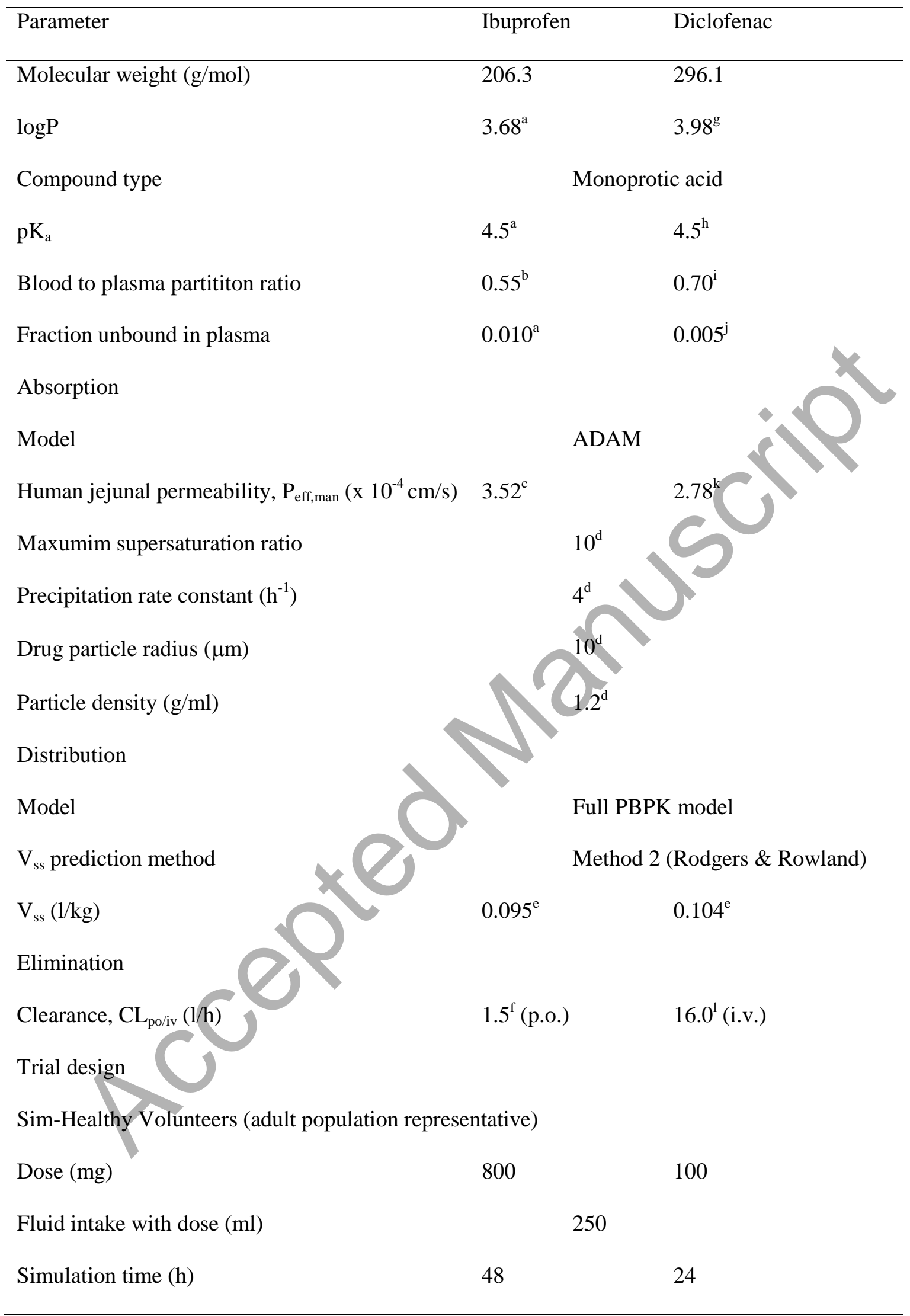

${ }^{\mathrm{a}}$ Taken from (Kasim et al. 2003); ${ }^{\mathrm{b}}$ Taken from (Obach et al. 1999); ${ }^{\mathrm{c}}$ Taken from (Beloica et al. 2015); ${ }^{\text {d }}$ Simcyp default; ${ }^{\text {e }}$ Simcyp predicted; ${ }^{\mathrm{f}}$ Optimized value; ${ }^{\mathrm{g}}$ Simcyp calculated 
(based on $\log \mathrm{D}_{6,8} 1.4$ (Chuasuwan et al. 2009) and $\mathrm{pK}_{\mathrm{a}} 4.5$ (Pobudkowska \& Domańska 2014)); ${ }^{\mathrm{h}}$ Taken from (Pobudkowska \& Domanska 2014); ${ }^{\mathrm{i}}$ Taken from (Tang et al. 1999);

${ }^{\mathrm{j}}$ Taken from (Kumar et al. 2002); ${ }^{\mathrm{k}}$ Simcyp calculated (based on Caco-2 permeability data (Yazdanian et al. 2004)); ${ }^{1}$ Taken from (Willis et al. 1979) 
Table 3. Solubility of the model drugs in different media.

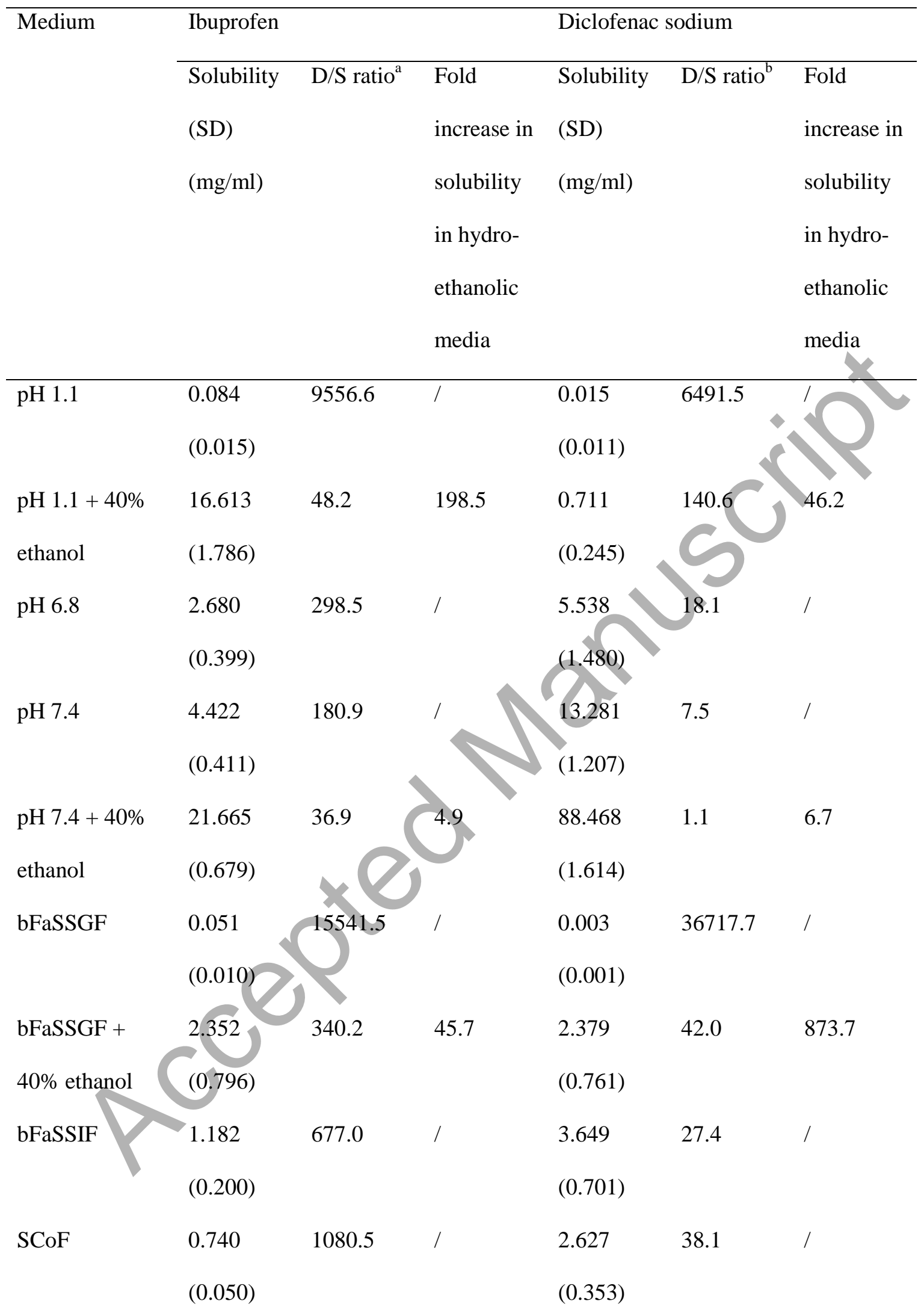

${ }^{a}$ Dose-solubility ratio for $800 \mathrm{mg}$ ibuprofen dose; ${ }^{\mathrm{b}}$ Dose-solubility ratio for $100 \mathrm{mg}$ diclofenacsodium dose 
Table 4. Comparison of the predicted and observed pharmacokinetic parameters for 800 mg IBU and $100 \mathrm{mg}$ DS SR tablets.

\begin{tabular}{|c|c|c|c|c|}
\hline & Parameter & $\mathrm{C}_{\max }(\mu \mathrm{g} / \mathrm{ml})$ & $\mathrm{t}_{\max }(\mathrm{h})$ & $\mathrm{AUC}_{0-\mathrm{t}}(\mu \mathrm{g} \mathrm{h} / \mathrm{ml})$ \\
\hline \multirow[t]{3}{*}{ Ibuprofen } & Predicted IBU1 (\%PE) & $12.35(9.52)$ & $17.28(-23.43)$ & $262.73(2.78)$ \\
\hline & Predicted IBU2 (\%PE) & $17.32(-26.89)$ & $14.16(-1.14)$ & $290.46(-7.48)$ \\
\hline & In vivo mean ${ }^{\mathrm{a}}$ & 13.65 & 14.00 & 270.24 \\
\hline \multirow[t]{4}{*}{ Diclofenac } & Predicted DS1 (\%PE) & $0.56(-1.82)$ & $2.16(13.16)$ & $3.83(23.25)$ \\
\hline & Predicted DS2 (\%PE) & $1.71(-210.91)$ & $2.40(4.00)$ & $4.59(8.02)$ \\
\hline & In vivo mean ${ }^{\mathrm{a}}$ & 0.55 & 2.50 & 4.99 \\
\hline & In vivo range ${ }^{c}$ & $0.41-4.12$ & $1.25-7.40$ & \\
\hline
\end{tabular}

${ }^{\mathrm{a}}$ Refer to the mean plasma concentration-time profile from a single study (Pargal et al. 1996); ${ }^{\mathrm{b}}$ Refer to the mean plasma concentration-time profile from a single study (Rao et al. 2001); ${ }^{\mathrm{c}}$ Refer to the range of mean values from different clinical studies (Davies \& Anderson 1997) 
Table 5. Predicted pharmacokinetic parameters for IBU and DS SR tablets (based on dissolution data from setup $\mathrm{C}$ with the addition of ethanol), and comparison with the predicted data that correspond to drug dissolution in non-ethanolic media.

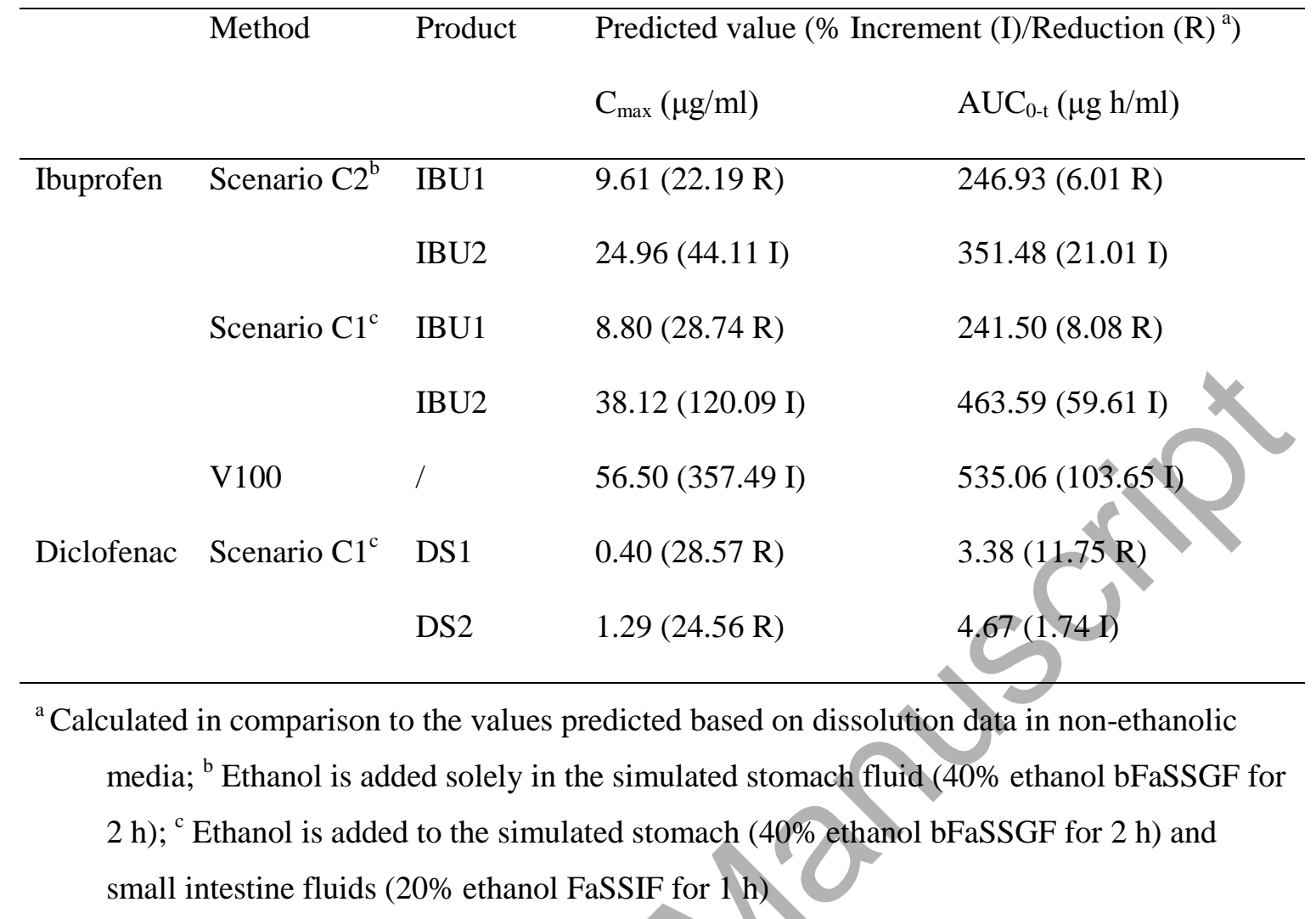

